\title{
LAS TÉCNICAS DE FERTILIZACIÓN ASISTIDA Y SUS EFECTOS EN EL DERECHO DE FILIACIÓN
}

\section{Evelyn I. Hervia Gutiérrez}

El presente artículo contiene un resumen, de la memoria de grado que presenté durante el año 2000 para optar al grado acadé mico de Licenciado en Ciencias Jurídicas de la Universidad Católica del Norte sede Coquimbo y la que fue aprobada con nota siete durante el año acadé mico 2001.

La investigación fue dirigida por el profesor guía Sr. Jorge Fernández Stevenson. siendo profesor informante don Pablo Arriagada Díaz.

\section{I.-Estructura}

La inexistencia de bibliografía que recopilara el material doctrinario existente sobre la aplicación de las técnicas de procreación asistida en Chile con posterioridad a la introducción de la Ley 19.585 publicada el 26 de octubre de 1998, y la riqueza de las numerosas aristas que el asunto genera, motivaron una investigación destinada a prestar una orientación acabada de la materia que permita adquirir una visión integral de los medios y los efectos que causa en la actualidad la aplicación de técnicas de procreación asistida.

Se inicia este trabajo introduciéndonos en los conceptos básicos relacionados a la aplicación de técnicas de procreación humana asistida. Analizamos en el primer capítulo el vocablo concepción, los conceptos de fertilización y fecundación, el significado y alcance de las palabras esterilidad e infertilidad; y conocemos asimismo las distintas clases de técnicas de procreación asistida utilizadas en la actualidad. Dichos conceptos originados en las ciencias de la biología, como la anatomía y la genética, nos acompañan durante todo nuestro estudio y su delimitación permite la mayor comprensión de los hechos que serán objeto del análisis jurídico; de las argumentaciones a favor y en contra de la regulación de las técnicas de procreación humana asistida y de la legislación existente en la materia.

Los capítulos segundo y tercero del trabajo los hemos dedicado al análisis de la situación jurídica de los materiales ontogénicos para la procreación y del producto de esta, es así como el segundo capítulo de la memoria de grado se titula "Situación Jurídica de los Gametos" en él se tratan los principales argumentos filosóficos y jurídicos que dan lugar a la calificación jurídica de espermatozoides y óvulos y los principales actos jurídicos de que ellos son objeto. El capítulo tercero en tanto, trata del embrión, su estatus frente al derecho y la protección que éste le brinda, analizando un 
asunto de gran interés doctrinario como es, la cuestión de la personalidad del que está por nacer.

La parte segunda de este estudio corresponde a la más fecunda fuente de información sobre las técnicas de procreación asistida: El Derecho Comparado. En él visualizamos los inicios de la aplicación de las técnicas de procreación asistida en los seres humanos; las distintas formas en que a nivel internacional se han enfrentado los problemas que de ellas han derivado resultando enriquecedor poder apreciar las consecuencias y efectos de las decisiones adoptadas por los legisladores y gobernantes de los Estados, valorando de este modo la necesidad de legislar en un sentido u otro.

Finamente, la tercera parte de este trabajo contiene el análisis de la legislación existente en Chile en lo relativo a las técnicas de procreación humana asistida, y su incidencia en el derecho de filiación. Esta tercera parte consta de tres capítulos, el primero de ellos analiza la legislación vigente en nuestro país vinculada a las técnicas de procreación asistida. Al amparo las normas contenidas en la Constitución Política de la República, hemos estudiado el contexto de las reglas contenidas en nuestro Código Civil, en el Libro Noveno del Código Sanitario y su reglamento, y en la Directiva Ministerial contenida en la resolución exenta $N^{\circ} 1072$ dictada en el año 1985. El conjunto de estas normas configura el marco legal aplicable a la práctica de las técnicas de procreación asistida humana, y ha sido necesario en esta parte determinar con precisión el alcance, procedencia y vigencia de las normas pertinentes.

El segundo capítulo de esta tercera parte constituye un aporte valioso de la doctrina nacional al análisis de la procedencia de una ley que regule la aplicación de las técnicas de procreación asistida humana. En este capítulo, nos detenemos para dar una mirada crítica al Proyecto de Ley que regula los principios jurídicos y éticos de las técnicas de Reproducción Humana Asistidas y establece sanciones para los infractores de sus normas.

Con todo, el entramado lógico de este recorrido por el estudio de las técnicas de procreación humana asistida y sus efectos en el derecho de filiación nos llevará a buscar la solución al principal problema planteado en el ámbito del Derecho Civil por la utilización de estas técnicas, cual es la determinación de la filiación de los hijos concebidos mediante ellas. Es precisamente este el asunto de que trata el capítulo final y para el cual nos hemos valido de toda la información recopilada en el transcurso de la memoria de grado en comento. No obstante el arduo trabajo de interpretación que ha requerido este capítulo final creemos que es mas bien un aporte a la discusión y análisis doctrinario futuro, que una respuesta definitiva a los problemas suscitados por la aplicación de las técnicas en el Derecho Civil. 


\section{II.- Forma de abordar el problema}

La única norma especial relativa a la filiación de los hijos nacidos mediante la aplicación de técnicas de procreación asistida es el nuevo artículo 182 del Código Civil, incorporado con ocasión de la reforma introducida por la Ley 19.585 publicada en Diario Oficial de 26 de octubre de 1998.

La nueva norma, vino a zanjar el problema de la determinación de la filiación de los hijos nacidos mediante la aplicación técnicas de reproducción asistida y las graves consecuencias que la falta de regulación ocasionaba.

Para abordar los problemas que suscitaba el empleo de la fecundación extracorporal, en lo que decía relación con el régimen jurídico de la filiación en el derecho nacional antes de la introducción del nuevo artículo 182 del Código Civil por la ley 19.585, era necesario elaborar en forma doctrinaria ciertas hipótesis lo que permitía, auque con gran dificultad y poca precisión, encuadrar los hechos en el marco legal existente.

Actualmente, no es necesario establecer en forma doctrinaria dichas hipótesis, puesto que la ley establece una hipótesis general, contenida en la presunción de paternidad y maternidad del artículo 182 el que dispone: "El padre y la madre del hijo concebido mediante las técnicas de reproducción humana asistida son el hombre y la mujer que se sometieron a ellas.

No podrá impugnarse la filiación determinada de acuerdo a la regla precedente, ni reclamarse una distinta."

El artículo 182 del Código Civil contiene una presunción de derecho que se configura mediante una estructura lógica de dos premisas, el razonamiento de la ley y finalmente, una conclusión. La primera premisa antecedente versa: "esta mujer y este hombre son los sometidos a las técnicas de reproducción humana asistida"; le sigue una segunda premisa cuyo contenido es "este hijo es concebido mediante la aplicación de la técnica de procreación asistida humana"; en tercer lugar la presunción contiene el razonamiento que afirma que "son padre y madre del hijo concebido mediante técnicas de procreación humana asistida el hombre y la mujer que se sometieron a las técnicas".

En la medida que seamos capaces de determinar el alcance y sentido de la hipótesis establecida por la ley, fijando el contenido de cada uno de sus elementos, será posible establecer quienes son los destinatarios de estas técnicas, y por lo tanto los sujetos de derecho a quienes se aplica la norma. En definitiva ello permitirá aplicar la presunción de derecho a cada caso concreto, determinando legalmente la filiación del concebido mediante la aplicación de técnicas de procreación artificial. 
Por su parte cuando, por faltar alguno de los elementos contenidos en la hipótesis general establecida por la ley, no pueda aplicarse la presunción al caso concreto, habrá que acudir a las normas generales sobre determinación de la filiación donde cobrará plena vigencia el principio de libre investigación. No obstante, no tenemos dudas de la posibilidad que se generen casos donde ni la norma especial del artículo 182 ni las normas generales del Código Civil sirvan para determinar la filiación.

Nos preguntaremos en primer lugar quienes son los sometidos a las técnicas de procreación humana asistida. El inciso primero del artículo 182 formula la hipótesis general en los siguientes términos: "El padre y la madre del hijo concebido mediante a la aplicación de técnicas de reproducción humana asistida son el hombre y la mujer que se sometieron a ella." Los sometidos a las técnicas solo podrán ser un hombre y una mujer estén casados o no. El legislador ha querido dejar claro el sexo de las personas sometidas a la técnica de procreación asistida, la exigencia, en correspondencia y armonía con el artículo 102 del Código Civil en lo relativo al matrimonio, para no dar lugar a equívoco en la interpretación de la norma. Pretende, recogiendo la experiencia legislativa extranjera, evitar que parejas de homosexuales y lesbianas puedan acceder a un hijo mediante la aplicación de las técnicas existentes pero también de técnicas futuras cuyo alcance desconocemos y que podrían vulnerar las leyes de la naturaleza en cuanto a que el hombre es padre y la mujer madre. La pareja heterosexual, como tal, es destinatario exclusivo de las técnicas de procreación artificial.

En segundo término, el efecto propio de la norma es generar un vínculo jurídico entre el padre y la madre con el hijo concebido mediante la técnica de procreación asistida, y brindar a ese hijo una situación jurídica más benéfica (la que solo se daría en el seno de una pareja) de la que tendría si la norma no existiese, ello en virtud del principio de interés superior del menor que debe tenerse en cuenta al interpretar cada letra de la ley. En consecuencia, conduciría al absurdo una interpretación que fuera lesiva para el menor, en que solo se tuviera en vista un interés unilateral del padre o de la madre por procrear.

Finalmente, creemos que de acuerdo el espíritu general de la legislación y la equidad natural, el mínimo jurídicamente exigido debe ser la pareja, lo que excluye a la mujer sola como sujeto de la aplicación de las técnicas. Estimamos acertada esta posición puesto que si bien de hecho muchas mujeres asumen solas su maternidad, nuestro Derecho no puede amparar esta situación ya que los principios constitucionales que le sirven de marco declaran como núcleo fundamental de la sociedad a la familia (Art. $1^{\circ}$ inciso $2^{\circ}$ ).

La pareja heterosexual que se somete a las técnicas de procreación asistica puede estar unida por vínculo matrimonial o no. El principio a generale sensu supone que donde la Ley no distingue no es lícito al intérprete distinguir, según esta máxima 
jurídica no podemos exigir mayores requisitos a la pareja heterosexual que los ya mencionados. Por tanto, bastará que la pareja se encuentre unida de hecho para que pueda acceder a la aplicación de la técnica de procreación asistida. Sin perjuicio de ello nos parece que la pareja unida por vínculo matrimonial cuya convivencia prolongada en el tiempo, no ha generado descendencia ofrece la mayor seguridad y bienestar al concebido.

El hombre y la mujer que se sometan a la técnica de procreación asistida serán en definitiva el padre y la madre del concebido mediante las técnicas de procreación humana asistida. Será necesario pues desentrañar el sentido del vocablo "someter" para que esté completo el entramado lógico que da lugar a la conclusión.

La palabra "someterse" la entendemos como la sumisión de la voluntad propia a la voluntad de otro, así se encuentra definido como "subordinar el juicio, decisión o afecto propios a los de otro" ${ }^{11}$, en ese sentido se somete a la técnica de procreación humana asistida quien consiente en su realización y este consentimiento emitido conjuntamente por los miembros de la pareja genera un vínculo contractual con el centro médico del cual se derivan derechos y obligaciones. Los efectos de la norma así interpretada nos llevan a distinguir entre dos contratos que concurren ante la realización de las técnicas de procreación asistida, el Contrato de Donación de Gametos y el contrato por el cual la pareja accede a la aplicación de la técnica que denominaremos Contrato de Aplicación de Técnica de Procreación Humana Asistida. En el contrato de aplicación de técnica de procreación asistida las partes serán el centro médico a cuyo equipo médico le corresponda practicar la técnica de procreación asistida ya sea que este mismo centro u otro haya tenido a su cargo el depósito de lo donado y la pareja compuesta por un hombre y una mujer que cumpliendo con los requisitos establecidos en la Directiva N 1072 del Ministerio de Salud consientan en la aplicación de la técnica.

Esta distinción tiene por objeto dejar claro que los sometidos a las técnicas de procreación asistida serán única y exclusivamente la pareja que es parte en el contrato de aplicación de técnica de procreación asistida puesto que el o la donante no se someten a la aplicación de una técnica de procreación humana asistida según el significado que le hemos atribuido a la palabra, sino a un contrato de donación celebrado con el centro médico.

\footnotetext{
'Diccionario de la Real Academia Española.
} 


\section{III.- A Modo de Conclusión}

Creemos que ha sido objetivo fundamental de este trabajo, posibilitar la comprensión de un hecho esencial en la vida de millones de seres humanos, la procreación, y llevarla al plano del derecho revestida de sus problemas y consecuencias.

Las técnicas de reproducción asistida son procedimientos médicos complejos, que sin subvertir el proceso de procreación natural ${ }^{2}$ o normal, intervienen en él de tal forma que coadyuvan a su resultado, superando las barreras naturales que impiden la realización de cada una de las etapas necesarias para dar origen a una vida humana desde la concepción hasta el nacimiento.

En una pareja fértil, este proceso procreativo, no reviste mayor complejidad que aquella que la misma naturaleza del acto requiere, sin embargo, en las parejas estériles la procreación del hijo se convierte en una búsqueda dramática por colmar el legítimo deseo de trascendencia y de culminación de la unión afectiva de hombre y mujer en el hijo.

Partiendo del sustrato básico enunciado hemos establecido como finalidad fundamental de las técnicas de procreación asistida que parejas estériles puedan tener hijos ya sea que entre ellas y sus hijos exista un vínculo biológico o no.

Sin embargo, creemos que la disociación entre paternidad y vínculo biológico, es un hecho que supera la condición natural del hombre, y que la intervención humana en la procreación mediante técnicas con intervención de terceros donantes no deben ser admitidas puesto que vulneran las leyes de la naturaleza y genera conflictos jurídicos y éticos de magnitud insospechada. La intervención de terceros donantes hace ilusoria la verdadera paternidad buscada por las técnicas de procreación asistida, y es perfectamente posible suplir el uso de una técnica de procreación asistida heteróloga por el medio que dispone la Ley al efecto, la adopción.

En razón de lo expresado creemos que resulta vital que toda permisividad respecto de las técnicas de procreación asistida tenga su punto de partida en un principio orientador básico fundado en que el hijo deseado por la pareja que se somete a una técnica de procreación asistida no puede ser nunca valorado como un objeto sino que debe ser valorado como un ser humano sujeto de derechos y detentador de una

\footnotetext{
2 "Nota de la autora, Usamos el vocablo "natural" como contrapuesto al término "artificial", sin embargo tal como se ha podido percibir en este trabajo hemos preferido hablar de procreación asistida y no de procreación artificial, porque creemos que no existe procreación artificial sino que esta será siempre un hecho de la naturaleza, no obstante exista una intervención de terceros ajenos a la pareja pero el instante mismo de la fecundación, de la unión de los gametos y de su material genético es un don que no depende de la ciencia.
} 
dignidad que le es propia por el hecho de ser hombre y que el ordenamiento jurídico no hace sino reconocer.

Es por eso que si bien creemos que debe legislarse en la materia, toda legislación debe ser fruto de un análisis dedicado, exhaustivo y conciente en donde participe toda la sociedad representada en sus distintos estamentos, puesto que el bien jurídico en juego, la vida del que está por nacer, es invaluable y que en última instancia, puede verse afectado un bien jurídico colectivo de relevancia presente y futura cual es EI Patrimonio Genético de la Humanidad.

El Derecho Nacional ha dado una primera respuesta al conflicto suscitado por las técnicas de procreación humana asistidas regulando la determinación de la filiación de los hijos concebidos mediante ellas. En adelante, es labor de los hombres y mujeres de Derecho construir una interpretación acorde con la dignidad del ser humano que reconoce nuestra Carta Fundamental, y basada como ella, en los principios del lus Naturalismo moderno. El Derecho de Familia está cambiando, y el Nuevo Derecho de Familia hace necesario una legislación contextualizada e imbuida de los principios de igualdad; libre investigación, y protección de los intereses del menor; conforme a los principios que establecen los Tratados sobre Derechos Humanos, pero por sobre todo que resguarde los intereses de la familia, como célula básica de la sociedad. 